\title{
HOMOGENEOUS LIE ALGEBRAS AND EXPANDING AUTOMORPHISMS
}

\author{
R. WARREN JOHNSON
}

ABSTRACT. A condition is given which, if satisfied by the spectrum of an automorphism of a Lie algebra, implies that the Lie algebra is homogeneous. In particular, almost all Lie algebras admitting expanding automorphisms are homogeneous.

1. Introduction. The question of whether all nilpotent Lie algebras admit expanding automorphisms (semisimple with all proper values of absoo lute value greater than 1) was answered by J. L. Dyer [3] with an example of a nilpotent Lie algebra which admits only unipotent automorphisms. In the same paper there is a theorem which states that all homogeneous Lie algebras (see definitions below) admit expanding automorphisms. In this note we will show that the converse, although false, is "almost" true. In fact, if a Lie algebra admits a grading automorphis $m$ it must be homogeneous. This has an interesting application to Anosov automorphisms and raises a problem about them. Suppose that $\Gamma$ is a discrete uniform subgroup of the nilpotent Lie group $M$ and that $\alpha$ is an $A$ nos ov automorphism of $M$ with respect to $\Gamma$ ( $\alpha$ re stricted to $\Gamma$ is an automorphism of $\Gamma$, and the differential of $\alpha$ at the identity has no proper values of absolute value 1 (see [2])). If $\alpha$ is grading, then $M$ admits expanding automorphisms. There is a rational Lie group $M_{Q}$ such that $\Gamma \subset M_{\mathrm{Q}} \subset M$ (see [1]). The unsolved question is: Do any of the expanding automorphisms leave $M_{\mathrm{Q}}$ invariant?

Homogeneous Lie algebras have also arisen in a different context in the work of G. Leger [6], where they are called quasi-cyclic nilpotent Lie algebras.

2. Definitions and notation. All unexplained notation and terminology is standard, as in [5] and [8]. All Lie a lgebras are defined over the rea ls, $R$,

Received by the editors December 12, 1973.

AMS (MOS) subject classifications (1970). Primary 17B30, 22E 25.

Key words and phrases. Nilpotent Lie group, nilpotent Lie algebra, homogeneous Lie algebra, quasi-cyclic nilpotent Lie algebra, expanding automorphism, Anosov automorphism. 
and, except for the free ones, are finite dimensional. For $S$ a subspace of a Lie algebra let $S^{i}$ be defined for $i$ a positive integer by $S^{1}=S$ and $S^{i+1}=$ $\left[S, S^{i}\right]$. If $\phi$ is a linear transformation let $\operatorname{spec}(\phi)$ denote its spectrum, and if $K$ is a $\phi$-invariant subspace let $\left.\phi\right|_{K}$ denote the restriction of $\phi$ to $K$.

Let $\mathfrak{\Omega}(X)$ denote the free Lie algebra on a set $X$. We shall only be concerned with the case $\operatorname{card}(X)=r$ finite, and we then say $\Omega(X)$ has rank $r$. We recall some standard facts about $\Omega(X)$. $\Omega(X)$ is an $\mathrm{N}$-graded algebra with $i$ th homogeneous component $\mathfrak{R}(X)_{i}$ and has for a bas is the image of a $\mathrm{P}$. Hall family on $X$, say $H_{i, j}$, where $H_{i, j} \in\left\{(X)_{i}\right.$ and $1 \leq j \leq \operatorname{dim} \Omega(X)_{i}$ (see [8]). The $H_{i, j}$ may be described as "nonassociative" monomials in $X=$ $\left\{X_{1}, X_{2}, \cdots, X_{r}\right\}$. We will need the fact that for $i>1, X_{k}$ cannot appear more than $i-1$ times in any $H_{i, j}$.

Let $c \Re(X)=\Omega(X) / \Omega(X)^{c+1}$ be the free nilpotent Lie algebra of class $c$ on $X$ with rank $=\operatorname{card}(X)$. In the induced grading, $c \Re(X)$ is an $\mathbf{N}$-graded algebra with $i$ th homogeneous component $\Re(X)_{i}$. The images of $H_{i, j}$, which we also denote by $H_{i, j}$, form a basis of homogeneous elements. If $M$ is a nilpotent Lie algebra of class $c$ and $\left(x_{1}, x_{2}, \cdots, x_{r}\right)$ is a basis for a complement $K$ to $\mathfrak{M}^{2}$ in $\mathbb{M}$, then there is a unique projection $\pi:{ }^{c} \Re(X) \rightarrow \mathfrak{M}$ with $X=$ $\left\{X_{1}, X_{2}, \cdots, X_{r}\right\}$ and $\pi\left(X_{k}\right)=x_{k}$. We will say $M$ is homogeneous if $\operatorname{ker} \pi$ is a homogeneous ideal in the graded structure on $c \Re(X)$. This notion clearly does not depend on the choice of either a complement or its basis in $M$. This definition is equivalent to that given in [3] and that of quasi-cyclic nilpotent Lie algebra in [6].

We will also need the following multinomial notation:

$$
\begin{array}{ll}
\mathbf{e}=\left(e_{1}, e_{2}, \cdots, e_{k}\right) \in \mathbf{N}^{k} ; & |\mathbf{e}|=e_{1}+e_{2}+\cdots+e_{k} ; \\
\lambda=\left(\lambda_{1}, \lambda_{2}, \cdots, \lambda_{k}\right) \in\left(\mathbf{R}_{+}^{*}\right)^{k} ; & \lambda^{\mathbf{e}}=\lambda_{1}^{e} 1 \cdot \lambda_{2}^{e} 2 \cdot \ldots \cdot \lambda_{k}^{e} .
\end{array}
$$

3. The condition. Let $\phi$ be an automorphism of a Lie algebra $M$. Since the group of automorphisms of $M$ is algebraic, the positive part of $\phi,|\phi|$, is also an automorphism [7]. Let us call $\operatorname{spec}(|\phi|)$ the positive spectrum of $\phi$. Since $|\phi|$ is semisimple, there is a $|\phi|$-invariant complement $K$ to $\mathfrak{M}^{2}$ in $\mathbb{M}$. Let us call $\operatorname{spec}\left(\left.|\phi|\right|_{K}\right)$ the restricted positive spectrum of $\phi$. If $\phi$ is an automorphism of the Lie algebra $M$ whose positive spectrum does not contain 1 , then $M$ is nilpotent (see [4]).

Let $\phi$ be an automorphism of the nilpotent Lie algebra $M$, and let $\left\{\lambda_{1}, \lambda_{2}, \cdots, \lambda_{k}\right\}$ be the restricted positive spectrum of $\phi$. We say that $\phi$ is grading if $\mathbf{e}, \mathbf{f} \in \mathbf{N}^{k}, 1<|\mathbf{e}|,|\mathbf{f}| \leq c=$ class of $\mathfrak{M}$, and $\lambda^{\mathbf{e}}=\lambda^{\mathbf{f}}$ implies $|\mathbf{e}|=|\mathbf{f}|$. 
Theorem. Let $\mathbb{M}$ be a nilpotent Lie algebra. $\mathbb{M}$ is homogeneous if and only if $M$ admits a grading automorphism.

Proof. That a homogeneous Lie algebra admits a grading automorphism is proved in J. L. Dyer [3] and G. Leger [6] in somewhat different terms. For the sake of completeness we prove it here. Let $M$ be a homogeneous Lie algebra of class $c$ and $\pi: c \Re(X) \rightarrow M$ some appropriate projection。 Write $\Re$ for $\operatorname{c\Re }(X)$. Let $\phi_{\lambda}, \lambda \neq 1, \lambda>0$, be the automorphism of $\Re$ which is scalar multiplication by $\lambda$ in $\Re_{1}$. Since $\operatorname{ker} \pi$ is homogeneous and $\phi_{\lambda}$ is scalar multiplication by $\lambda^{i}$ in $\Re_{i}$, ker $\pi$ is $\phi_{\lambda}$-invariant and the induced automorphism in $M$ has restricted positive spectrum $\{\lambda\}$. But then, since an automorphism with restricted positive spectrum $\{\lambda\}$ with $\lambda \neq 1$ is grading for any Lie algebra, we are done.

Conversely, suppose $M$ admits a grading a utomorphism $\phi$, where $\mathscr{M}$ is nilpotent of class $c_{0}$. Let $K$ be a $|\phi|$-invariant complement to $\mathfrak{M}^{2}$ in $M$ and $K=\bigoplus_{i=1}^{k} K_{\lambda_{i}}$ be the primary decomposition of $K$ relative to $\left.|\phi|\right|_{K}$. Choose $\left(x_{1}, \cdots, x_{n_{1}}, x_{n_{1}+1}, \cdots, x_{n_{2}}, \cdots, x_{n_{k}}\right)$ to be a basis of $K$ compatible with this decomposition (i.e., $\left(x_{n_{i-1}+1}, \cdots, x_{n_{i}}\right)$ is a basis of $K_{\lambda_{i}}$ with $n_{0}=0$ ). Let

$$
X=\left\{X_{1}, \cdots, X_{n_{1}}, X_{n_{1}+1}, \cdots, X_{n_{2}}, \cdots, X_{n_{k}}\right\}
$$

and $\pi:{ }^{c} \Re(X) \rightarrow M$ be the projection which satisfies $\pi\left(X_{i}\right)=x_{i}$. We will show that ker $\pi$ is homogeneous when $\phi$ is grading.

In fact, let $\mathfrak{M}^{2}=\bigoplus M_{\mu}^{2}$ be the primary decomposition of $\mathfrak{M}^{2}$ relative to $\left.|\phi|\right|_{\mathfrak{D}^{2}}$. Now for each proper value $\mu$ there is an $\mathbf{e} \in \mathbf{N}^{k}$ with $1<|\mathbf{e}| \leq c$ such that $\mu=\lambda^{\mathrm{e}}$, since $K$ generates $\mathscr{M}$. Moreover, since $\phi$ is grading, $|\mathbf{e}|$ is unique, and we can define $|\mu|=|\mathbf{e}|$. Then if $\left\{P_{\mu}\right\}$ is the set of projections corre. sponding to $\mathscr{M}^{2}=\bigoplus M_{\mu}^{2},\left\{P_{\mu}\right\}$ is partitioned by the sets $\left\{P_{\nu}\right\}_{|\nu|=j}$, where $j=2, \cdots, c$. Then $\left\{P_{(j)}=\bigoplus_{|\nu|=j} P_{\nu}\right\}$ for $j=2, \cdots, c$ is a supplementary set of orthogonal projections inducing, if $\mathbb{M}_{(j)}^{2}$ is the image of $P_{(j)}$, the decomposition $\mathfrak{M}^{2}=\bigoplus_{j=2}^{c} \mathfrak{M}^{2}(j)^{\bullet}$

Since $c$ and $X$ are fixed, write $\Re$ for ${ }^{c} \Re(X)$. Because $\pi\left(\Re_{1}\right)=K$, in order for ker $\pi$ to be homogeneous it is sufficient to show that for all $Y \in \Re^{2}$, with $Y=Y_{2}+Y_{3}+\cdots+Y_{c}$ its decomposition into homogeneous components, that $\pi(Y)=0$ implies $\pi\left(Y_{j}\right)=0$. To this end let $Y_{j}=\Sigma a_{j, l} H_{j, l}$ be the representation of $Y_{j}$ in terms of a P. Hall basis, $H_{j, l}$, of $\Re_{j}$. 
But, $|\phi|\left(\pi\left(H_{j, l}\right)\right)=\lambda^{\mathbf{e}}{ }_{j, l} \pi\left(H_{j, l}\right)$, where $\left|\mathbf{e}_{j, l}\right|=j$. Now either $\lambda^{\mathbf{e}_{j, l}}=\nu \epsilon$ $\operatorname{spec}\left(\left.|\phi|\right|_{\mathbb{R}^{2}}\right)$ and, hence, $\pi\left(H_{j, l}\right) \in \mathfrak{M}_{\nu}^{2} \subset \mathfrak{M}_{(j)}^{2}$, or $\lambda^{e}{ }^{e}, \notin \notin \operatorname{spec}\left(\left.|\phi|\right|_{\mathbb{R}^{2}}\right)$ and $\pi\left(H_{j, l}\right)=0 \in \mathbb{M}_{(j)}^{2}$. Hence $\pi\left(Y_{j}\right) \in \mathbb{M}_{(j)}^{2}$, and since $\mathbb{M}^{2}=\bigoplus_{(j)}^{2}, \pi(Y)=0 \mathrm{im}$ plies $\pi\left(Y_{j}\right)=0$. We have finished the proof.

Corollary 1 (G. Leger [6]). A nilpotent Lie algebra $M$ is homogeneous if and only if there exists a complement $K$ to $\mathfrak{M}^{2}$ in $M$ such that the identity map on $K$ extends to a derivation of $M$.

Corollary 2. If a nilpotent Lie algebra admits an automorphism whose restricted positive spectrum $\left\{\lambda_{1}, \cdots, \lambda_{k}\right\}$ has the property that $\left\{\log \lambda_{1}, \cdots, \log \lambda_{k}\right\}$ is Q-independent, then it is homogeneous.

Proof. Such an automorphism is grading for any nilpotent Lie algebra.

4. Some examples. The 5-dimensional nilpotent Lie algebra with bas is $\left(x_{1}, \cdots, x_{5}\right)$ and relations

$$
\left[x_{1}, x_{2}\right]=x_{3}, \quad\left[x_{1}, x_{3}\right]=x_{4}, \quad\left[x_{1}, x_{4}\right]=\left[x_{2}, x_{3}\right]=x_{5},
$$

is not homogeneous (for $\lambda \neq 1, x_{1} \mapsto \lambda x_{1}, x_{2} \mapsto \lambda x_{2}$ does not extend to an automorphis $\mathrm{m}$ ), but does admit an expanding automorphism extending $x_{1} \mapsto$ $\lambda x_{1}, x_{2} \mapsto \lambda^{2} x_{2}$ for $\lambda>1$.

This example can be extended as follows: if for $\left\{\lambda_{1}, \cdots, \lambda_{k}\right\} \subset \mathbf{R}_{+}^{*}$, and $1<|\mathbf{e}|<|\mathbf{f}|=c, \lambda^{\mathbf{e}}=\lambda^{\mathbf{f}}$, then there exists a nilpotent Lie algebra of class $c$ and rank $\leq k+1$ which is not homogeneous but does admit an automorphism whose restricted positive spectrum is $\left\{\lambda_{1}, \cdots, \lambda_{k}\right\}$.

\section{REFERENCES}

1. L. Auslander, An exposition of the structure of solvmanifolds. I: Algebraic theory, Bull. Amer. Math. Soc. 79 (1973), $227-261$.

2. L. Auslander and J. Scheuneman, On certain automorphisms of nilpotent Lie groups, Proc. Sympos. Pure Math., vol. 14, Amer. Math. Soc., Providence, R. I., 1970, pp. 9-15. MR 42 \#5284.

3. J. L. Dyer, A nilpotent Lie algebra with nilpotent automorphism group, Bull. Amer. Math. Soc. 76 (1970), 52-56. MR 40 \#2789.

4. N. Jacobson, $A$ note on automorphisms and derivations of Lie algebras, Proc. Amer. Math. Soc. 6 (1955), 281-283. MR 16, 897.

5. - Lie algebras, Interscience Tracts in Pure and Appl. Math., no. 10, Interscience, New York, 1962. MR 26 \#1345.

6. G. Leger, Derivations of Lie algebras. III, Duke Math J. 30 (1963), 637-645. MR $28 \# 3064$. 
7. G. D. Mostow, Factor spaces of solvable groups, Ann. of Math. (2) 60 (1954), 1-27. MR 15, 853.

8. J.-P. Serre, Lie algebras and Lie groups, Benjamin, New York, 1965. MR $36 \# 1582$.

DE PARTMENT OF MATHEMATICS, LEHIGH UNIVERSITY, BETHLEHEM, PENNSYLVANIA 18015

Current address: Department of Mathematics and Computer Science, St. Cloud State College, St. Cloud, Minnesota 56301 